\title{
Dynamic Optimization with a Nonsmooth, Nonconvex Technology: The Case of a Linear Objective Function
}

\author{
Takashi Kamihigashi* \\ RIEB \\ Kobe University \\ tkamihig@rieb.kobe-u.ac.jp
}

\author{
Santanu Roy \\ Department of Economics \\ Southern Methodist University \\ sroy@smu.edu
}

July 31,2004

\begin{abstract}
This paper studies a one-sector optimal growth model with linear utility in which the production function is generally nonconvex, nondifferentiable, and discontinuous. The model also allows for a general form of irreversible investment. We show that every optimal path either converges to zero or reaches a positive steady state in finite time (and possibly jumps among different steady states afterwards). We establish conditions for extinction (convergence to zero), survival (boundedness away from zero), and the existence of a minimum safe standard of conservation. They extend the conditions known for the case of S-shaped production functions to a much large class of technologies. We also show that as the discount factor approaches one, optimal paths converge to a small neighborhood of the golden rule capital stock.
\end{abstract}

Keywords: Nonconvex, nonsmooth, and discontinuous technology; extinction; survival; turnpike; linear utility.

${ }^{*}$ Corresponding author: RIEB, Kobe Univeristy, Rokkodai, Nada, Kobe 657-8501, Japan Phone/Fax: +81-78-803-7015, Email: tkamihig@rieb.kobe-u.ac.jp 


\section{Introduction}

The aggregative model of optimal economic growth is an important theoretical paradigm that is widely used for analyzing economic issues related to intertemporal allocation of resources and capital accumulation. Much of the existing literature focuses on "classical" versions of the model where the underlying technology is smooth and convex. Yet, there exist a large variety of economic settings where the technology exhibits nonconvexities, nonsmoothness, and even discontinuities due to various factors such as fixed costs, threshold effects, increasing returns to scale, economies of scope, and depensation in biological reproduction processes. This paper presents a comprehensive analysis of a one-sector optimal growth model with linear utility that admits nonconvex, nonsmooth, and discontinuous technology as well as irreversible investment. ${ }^{1}$

The literature on optimal growth with nonconvex technology dates back to Clark (1971), who analyzed the problem of optimal dynamic consumption of a biological resource where the production function is S-shaped and the objective function is linear in consumption. ${ }^{2}$ A full characterization of optimal paths in the context of optimal growth was provided by Majumdar and Mitra (1983), who showed among other things that if the discount factor is in an intermediate range, there exists a critical stock (minimum safe standard of conservation) that determines whether an optimal path converges to zero or to a positive steady state. Mitra and Ray (1984) studied a more general model with concave utility in which the production function is only required to be strictly increasing and continuous.

While various results are known on nonconvex one-sector optimal growth models with strictly concave utility (e.g., Skiba, 1978; Majumdar and Mitra, 1982; Majumdar and Nermuth, 1982; Dechert and Nishimura, 1983; Kamihigashi and Roy, 2003), ${ }^{3}$ many of the arguments used in the case of strictly concave utility cannot readily be applied to the linear utility case. For example, arguments based on the Euler equation cannot directly be applied to the linear utility case, where optimal paths are often not in the interior of the feasible set. In addition, though optimal paths are known to be monotone in

\footnotetext{
${ }^{1}$ The case of strictly concave utility is studied in Kamihigashi and Roy (2003). This paper mostly focuses on arguments specific to the linear utility case.

${ }^{2}$ Optimal growth models with convex technology and linear utility have been studied, among others, by Srinivasan (1964), Uzawa (1964), Malinvaud (1965), and Shell (1967).

${ }^{3}$ See Kamihigashi and Roy (2003) for a discussion of this literature.
} 
the case of strictly concave utility, this is not true in the linear utility case, as shown in Proposition 3.2 of this paper.

We show however that every optimal path is strictly monotone until it reaches a steady state. Furthermore, every optimal path either converges to zero or reaches a positive steady state in finite time (and possibly jumps among different steady states afterwards). This sharpens, in the linear utility case, the result by Mitra and Ray (1984) that every optimal path approaches the set of steady states asymptotically, and extends it to cases with discontinuous production functions and irreversible investment. We also establish conditions for extinction (convergence to zero), survival (boundedness away from zero), and the existence of a minimum safe standard of conservation. These conditions extend those established by Clark (1971) and Majumdar and Mitra (1983) in the case of S-shaped production functions to a much larger class of technologies.

Moreover we show that despite the nonclassical features of the model, as the discount factor approaches one, optimal paths converge to a small neighborhood of the golden rule capital stock. This result allows us to extend the turnpike theorem of Majundar and Nermuth (1982) to the case of linear utility. Though turnpike properties are normally associated with concavity (e.g., McKenzie, 1986), our model essentially involves no concavity.

Our analysis has a number of applications outside optimal economic growth. First, it can be used to study the optimal exploitation of renewable resources (e.g., Clark, 1971). Second, our model can be viewed as the problem of a firm that maximizes the discounted sum of retained profits (dividends), faces a constant rate of interest, and invests part of its current flow of net revenue that in turn determines next period's flow of net revenue. Third, many of the techniques developed in this paper can be applied to a wider class of models where the objective function is additively separable in current and future state variables (e.g., Spence and Starrett, 1975; Clark, 1990, Section 7.7).

The rest of the paper is organized as follows. Section 2 describes the model. Section 3 develops various properties that constitute the essential tools of our analysis. Section 4 shows results on monotonicity and convergence of optimal paths. Section 5 offers conditions for survival, extinction, and the existence of a minimum safe standard of conservation. Section 6 establishes turnpike properties of optimal paths. 


\section{The Model}

Consider the following maximization problem:

$$
\begin{aligned}
\max _{\left\{c_{t}, x_{t}\right\}_{t=0}^{\infty}} & \sum_{t=0}^{\infty} \delta^{t} c_{t} \\
\text { s.t. } & \forall t \in \mathbb{Z}_{+}, c_{t}+x_{t+1}=f\left(x_{t}\right), \\
& c_{t} \geq 0 \\
& x_{t+1} \geq r\left(x_{t}\right) \\
& x_{0} \text { given, }
\end{aligned}
$$

where $c_{t}$ is consumption in period $t, x_{t}$ is the capital stock at the beginning of period $t, \delta$ is the discount factor, $f$ is the production function, and (2.4) means that capital cannot be decreased below its depreciated level $r\left(x_{t}\right)$; we call $r$ the depreciation function for convenience. Our formulation allows for nonlinear depreciation. The standard case of reversible investment is a special case in which $r(x)=0$ for all $x \geq 0 .{ }^{4}$

We use the following standard definitions. A path $\left\{c_{t}, x_{t}\right\}_{t=0}^{\infty}$ is feasible if it satisfies (2.2)-(2.4). A capital path $\left\{x_{t}\right\}$ is feasible if there is a consumption path $\left\{c_{t}\right\}$ such that $\left\{c_{t}, x_{t}\right\}$ is feasible. A path from $x_{0}$ is a path $\left\{c_{t}^{\prime}, x_{t}^{\prime}\right\}$ such that $x_{0}^{\prime}=x_{0}$. A capital path from $x_{0}$ is defined similarly. A feasible path $\left\{c_{t}, x_{t}\right\}$ is optimal (from $x$ ) if it solves the maximization problem (2.1)-(2.5) (with $x_{0}=x$ ). A feasible capital path $\left\{x_{t}\right\}$ is optimal (from $x$ ) if there is a consumption path $\left\{c_{t}\right\}$ such that $\left\{c_{t}, x_{t}\right\}$ is optimal (from $x$ ). A stationary (capital) path is a constant feasible (capital) path. A capital stock $x \geq 0$ is a steady state if the stationary path from $x$ is optimal. The assumptions stated below are maintained throughout the paper. ${ }^{5}$

Assumption 2.1. $\delta \in(0,1)$.

Assumption 2.2. (i) $f: \mathbb{R}_{+} \rightarrow \mathbb{R}_{+}$is strictly increasing and upper semicontinuous. (ii) $f(0)=0$.

The production function $f$ is not required to be continuous or differentiable. To state our assumption on the depreciation function $r$, for $h: \mathbb{R}_{+} \rightarrow$ $\mathbb{R}$, we define

$$
h_{-}(x)=\lim _{y \uparrow x} h(y), \quad h_{+}(x)=\lim _{y \downarrow x} h(y),
$$

\footnotetext{
${ }^{4}$ See Kamihigashi and Roy (2003) for a more detailed discussion of (2.4).

${ }^{5}$ Assumptions 2.2 and 2.3 are discussed in more detail in Kamihigashi and Roy (2003).
} 
provided that the right-hand sides are well-defined.

Assumption 2.3. (i) $r: \mathbb{R}_{+} \rightarrow \mathbb{R}_{+}$is nondecreasing and lower semicontinuous. (ii) $\forall x>0, r_{+}(x)<x$ and $r(x)<f(x)$.

Like the production function $f$, the depreciation function $r$ is not required to be differentiable or continuous. Part (ii) means that the irreversibility constraint (2.4) is never binding at a steady state and that positive consumption is available from any positive initial capital stock. The following assumption rules out unbounded growth

Assumption 2.4. $\exists \hat{x}>0, \forall x>\hat{x}, f(x)<x$.

Remark 2.1. If $\exists x>0, f(x) \leq x$, then any feasible capital path $\left\{x_{t}\right\}$ from $x_{0} \in[0, x]$ satisfies $\forall t \in \mathbb{Z}_{+}, x_{t} \leq x$.

Assumption 2.4 together with Remark 2.1 implies that all feasible paths are bounded. We rule out unbounded growth since it does not seem to be a robust phenomenon with linear utility. ${ }^{6}$ The assumptions made above ensure the existence of an optimal path from any initial capital stock $x_{0} \geq 0$ by a standard argument (e.g., Ekeland and Scheinkman, 1986, Proposition 4.1).

\section{Useful Properties}

\subsection{The Value Function and Some Definitions}

This subsection establishes basic properties of the value function in addition to defining useful functions and correspondences. Let $v: \mathbb{R}_{+} \rightarrow \mathbb{R}_{+}$be the value function for the maximization problem (2.1)-(2.5). ${ }^{7}$ The Bellman equation is given by

$$
v(x)=\max _{y \in F(x)}\{f(x)-y+\delta v(y)\}
$$

\footnotetext{
${ }^{6}$ For example, suppose $f(x)=A x$ for some $A>0$ and $r(x)=0$ for all $x \geq 0$. Let $x_{0}>0$. Then it is easy to see that if $A<1 / \delta$, the unique optimal path is given by $x_{t+1}=0$ for all $t \geq 0$; if $A>1 / \delta$, no optimal path exists; and if $A=1 / \delta$, any feasible capital path satisfying $\lim _{T \uparrow \infty} \delta^{T} x_{T+1}=0$ is optimal. Hence unbounded growth is possible, but is expected only in a knife edge case even for a more general production function.

${ }^{7}$ Because of the irreversibility constraint (2.4), $v$ need not be nondecreasing, as discussed in Kamihigashi and Roy (2003, Section 3.1).
} 
where $F(x)=\{y \geq 0 \mid r(x) \leq y \leq f(x)\}$. Let $K$ denote the optimal policy correspondence. For $x \geq 0$, define

$$
\begin{aligned}
& g(x)=f(x)-x, \\
& \gamma(x)=\delta f(x)-x, \\
& \lambda(x)=\delta v(x)-x
\end{aligned}
$$

The function $g(x)$ gives the stationary level of consumption associated with the capital stock $x$. We call $\gamma(x)$ the partial gain function for the following reason. An investment of $x$ units of capital generates $f(x)$ units of output in the next period. Hence the "net gain" is $\delta f(x)-x$. This gain however is partial in the sense that it does not take account of future gains obtained by reinvestment. The "total net gain" is captured by $\lambda(x)$, which we call the total gain function.

Lemma 3.1. (i) $v$ is upper semicontinuous. (ii) $\forall x>0, v(x)>0$.

Proof. Part (i) can be shown by a standard argument (e.g., Kamihigashi and Roy, 2003, Lemma 3.3). To see (ii), let $\left\{c_{t}, x_{t}\right\}$ be the path from $x$ such that $\forall t \in \mathbb{Z}_{+}, x_{t+1}=r\left(x_{t}\right)$. Since this path is feasible and $\forall t \in \mathbb{Z}_{+}, c_{t} \geq 0$,

$$
v(x) \geq c_{0}=f(x)-r(x)>0,
$$

where the last inequality holds by Assumption 2.3(ii). Now (ii) holds.

\subsection{Properties Based on $\gamma(x)$}

This subsection shows some basic results using the partial gain function $\gamma(x)$. Our first result here reduces the maximization problem (2.1)-(2.5) to one in which the discounted sum of $\gamma\left(x_{t+1}\right)$ is to be maximized. This type of reduction was used by Spence and Starrett (1975) and Clark (1990) to study optimality of "most rapid approach" paths. We use it here for more general purposes.

Lemma 3.2. A feasible capital path $\left\{x_{t}\right\}$ is optimal iff it solves the following maximization problem:

$$
\max _{\left\{x_{t}\right\}_{t=0}^{\infty}} \sum_{t=0}^{\infty} \delta^{t} \gamma\left(x_{t+1}\right) \quad \text { s.t. } \forall t \in \mathbb{Z}_{t}, x_{t+1} \in F\left(x_{t}\right), x_{0} \text { given. }
$$


Proof. For any feasible capital path $\left\{x_{t}\right\}$,

$$
\begin{aligned}
& \sum_{t=0}^{\infty} \delta^{t}\left[f\left(x_{t}\right)-x_{t+1}\right] \\
= & f\left(x_{0}\right)-x_{1}+\delta f\left(x_{1}\right)-\delta x_{2}+\delta^{2} f\left(x_{2}\right)-\delta^{2} x_{3}+\cdots \\
= & f\left(x_{0}\right)+\sum_{t=0}^{\infty} \delta^{t} \gamma\left(x_{t+1}\right),
\end{aligned}
$$

where the last sum exists since $\left\{\gamma\left(x_{t+1}\right)\right\}$ is bounded by boundedness of $\left\{x_{t}\right\}$ and monotonicity of $f$. The lemma now follows.

Though Lemma 3.2 may seem rather obvious, it does not necessarily hold if Assumption 2.4 is dropped. ${ }^{8}$ The following result generalizes Mitra and Ray (1984, Theorem 4.2) in the case of linear utility. ${ }^{9}$

Proposition 3.1. (i) Any $\tilde{x} \in \operatorname{argmax}_{x \geq 0} \gamma(x)$ is a steady state. ${ }^{10}$ (ii) If $\exists y>0, \gamma(y) \geq 0$, then there exists a nonzero steady state.

Proof. Immediate from Lemma 3.2 and upper semicontinuity of $\gamma$.

Proposition 3.2. Let $x^{1}, x^{2}>0$ be steady sates such that $x^{1} \in F\left(x^{2}\right)$ and $x^{2} \in F\left(x^{1}\right)$. Then any capital path $\left\{x_{t}\right\}$ such that $\forall t \in \mathbb{Z}_{+}, x_{t} \in\left\{x^{1}, x^{2}\right\}$ is optimal.

Proof. Since $x^{2} \in F\left(x^{1}\right), \gamma\left(x^{2}\right) \leq \gamma\left(x^{1}\right)$ by Lemma 3.2. Likewise $\gamma\left(x^{1}\right) \leq$ $\gamma\left(x^{2}\right)$. Thus $\gamma\left(x^{1}\right)=\gamma\left(x^{2}\right)$. The conclusion now follows from Lemma 3.2.

By Proposition 3.1(i), the hypothesis of Proposition 3.2 holds if there are two maximizers $x^{1}, x^{2}$ of $\gamma(x)$ and if $x^{1} \in F\left(x^{2}\right)$ and $x^{2} \in F\left(x^{1}\right)$. In this case, cycles of any period as well as nonperiodic paths are possible by Proposition 3.2. The result can easily be extended to any number of steady states with more complicated transition patterns (e.g., Kamihigashi, 1999, Figure 2).

The following result is useful in determining the direction in which an optimal capital path possibly moves.

\footnotetext{
${ }^{8}$ For example, suppose $f(x)=x / \delta$. Then $\gamma(x)=0$ for all $x \geq 0$. Hence any feasible capital path trivially solves (3.6). In particular, the capital path $\left\{x_{t}\right\}$ from $x_{0}>0$ given by $x_{t+1}=f\left(x_{t}\right)$ for all $t \geq 0$ solves (3.6). But in this case, $c_{t}=f\left(x_{t}\right)-x_{t+1}=0$ for all $t \in \mathbb{Z}_{+}$, so this is in fact the worst feasible path.

${ }^{9}$ See Kamihigashi and Roy (2003, Propositions 3.3, 3.4) for the case of strictly concave utility.

${ }^{10}$ The maximum exists since $\gamma$ is upper semicontinuous and $\gamma(x)<0$ for $x>\hat{x}$, where $\hat{x}$ is given by Assumption 2.4 .
} 
Lemma 3.3. Let $\left\{x_{t}\right\}$ be an optimal capital path from $x_{0} \geq 0$. Then either (i) $\exists t \in \mathbb{Z}_{+}, \gamma\left(x_{t+1}\right)>\gamma\left(x_{0}\right)$ or (ii) $\forall t \in \mathbb{Z}_{+}, \gamma\left(x_{t+1}\right)=\gamma\left(x_{0}\right)$.

Proof. Suppose both cases (i) and (ii) are false. Then $\forall t \in \mathbb{Z}_{+}, \gamma\left(x_{t+1}\right) \leq$ $\gamma\left(x_{0}\right)$ and $\exists t \in \mathbb{Z}_{+}, \gamma\left(x_{t+1}\right)<\gamma\left(x_{0}\right)$. Thus

$$
0 \leq f\left(x_{0}\right)+\sum_{t=0}^{\infty} \delta^{t} \gamma\left(x_{t+1}\right)<f\left(x_{0}\right)+\frac{\gamma\left(x_{0}\right)}{1-\delta}=\frac{g\left(x_{0}\right)}{1-\delta}
$$

Hence the stationary path from $x_{0}$ is feasible. But then by the above strict inequality and Lemma 3.2, $\left\{x_{t}\right\}$ cannot be optimal, a contradiction.

Lemma 3.3 means that an optimal capital path must achieve a higher partial gain $\gamma\left(x_{t+1}\right)$ at some point unless partial gains are constant over time. In other words, it never moves in a direction in which only lower partial gains are available. ${ }^{11}$

\subsection{Properties Based on $\lambda(x)$}

This subsection shows some basic results using the total gain function $\lambda(x)$. The following result characterizes the policy correspondence by a single static maximization problem.

Lemma 3.4. Let $x, y \geq 0$. Then $y \in K(x)$ iff $y \in \operatorname{argmax}_{z \in F(x)} \lambda(z)$.

Proof. Note from (3.1) and (3.4) that

$$
v(x)=\max _{z \in F(x)}\{f(x)+\lambda(z)\}=f(x)+\max _{z \in F(x)} \lambda(z) .
$$

The conclusion now follows.

Corollary 3.1. $x \geq 0$ is a steady state iff $x \in \operatorname{argmax}_{z \in F(x)} \lambda(z)$.

Proof. Immediate from Lemma 3.4.

For $x \geq 0$, define

$$
\underline{K}(x)=\min \{y \mid y \in K(x)\}, \quad \bar{K}(x)=\max \{y \mid y \in K(x)\} .
$$

The above minimum and maximum exist since $K(x)$ is compact by Lemma 3.4 and upper semicontinuity of $\lambda$. The next result follows from Topkis (1978, Theorem 6.1), but can easily be shown by using the total gain function $\lambda$.

\footnotetext{
${ }^{11}$ A slightly different version of Lemma 3.3 holds in the case of strictly concave utility (Kamihigashi and Roy, 2003, Lemma 3.5).
} 
Lemma 3.5. (i) Let $x, y \geq 0, y \in K(x)$, and $y^{\prime} \in K\left(x^{\prime}\right)$. Then $\min \left\{y, y^{\prime}\right\} \in$ $K\left(\min \left\{x, x^{\prime}\right\}\right)$ and $\max \left\{y, y^{\prime}\right\} \in K\left(\max \left\{x, x^{\prime}\right\}\right)$. (ii) $\underline{K}$ and $\bar{K}$ are nondecreasing.

\subsection{Implications of Lemma 3.5}

This subsection shows two lemmas that require only the properties of $K$ stated in Lemma 3.5. The first one shows that an optimal capital path can change direction only at a steady state, and that if it changes direction, there is a "shortcut."

Lemma 3.6. Let $x \geq 0, y \in K(x)$, and $z \in K(y)$. Suppose (a) $x \leq y \geq z$ or (b) $x \geq y \leq z$. Then (i) $y$ is a steady state and (ii) $z \in K(x)$.

Proof. In case (a), $y=\max \{y, z\} \in K(\max \{x, y\})=K(y)$ and $z=\min \{y, z\} \in$ $K(\min \{x, y\})=K(x)$ by Lemma $3.5(\mathrm{i})$. Case (b) is similar.

The next result shows that two different capital stocks achieved along an optimal capital path can always be connected by a monotone path that is also optimal. It allows us to simplify some of our arguments.

Lemma 3.7. Let $n \geq 2$. Let

$$
x^{1} \geq 0, x^{2} \in K\left(x^{1}\right), \ldots, x^{n} \in K\left(x^{n-1}\right) .
$$

Then there exists a monotone (i.e., nondecreasing or nonincreasing) path $\left\{y^{i}\right\}_{i=1}^{m}$ with $m \geq 2$ such that $y^{1}=x^{1}, y^{m}=x^{n}$, and $y^{2} \in K\left(y^{1}\right), \ldots, y^{m} \in$ $K\left(y^{m-1}\right)$.

Proof. If $\left\{x^{i}\right\}_{i=1}^{n}$ is monotone, the conclusion is obvious. So suppose it is not monotone, which requires $n \geq 3$. Then $\exists j \in\{1, \ldots, n-2\}, x^{j} \leq x^{j+1} \geq x^{j+2}$ or $x^{j} \geq x^{j+1} \leq x^{j+2}$. By Lemma 3.6(ii), $x^{j+2} \in K\left(x^{j}\right)$. For $i=1, \ldots, n-1$, define $\tilde{x}^{i}=x^{i}$ if $i \leq j$ and $\tilde{x}^{i}=x^{i+1}$ if $i>j$ (i.e., remove $x^{j+2}$ ). Then $\left\{\tilde{x}^{i}\right\}_{i=1}^{n-1}$ satisfies (3.13) with appropriate modifications. If $\left\{\tilde{x}^{i}\right\}$ is not monotone, apply the same procedure to remove another element. The procedure can be applied at most $n-2$ times since each application removes one element. Since $x^{1}$ and $x^{n}$ are never removed, this process yields a desired monotone path. 


\subsection{Euler Inequalities}

For $h:(a, b) \rightarrow \mathbb{R}$ with $a<b$, define

$$
h_{-}^{\prime}(x)=\varliminf_{\epsilon \downarrow 0} \frac{h(x)-h(x-\epsilon)}{\epsilon}, \quad h_{+}^{\prime}(x)=\varlimsup_{\epsilon \downarrow 0} \frac{h(x+\epsilon)-h(x)}{\epsilon} .
$$

These generalized derivatives allow us to obtain "Euler inequalities" instead of an Euler equation. Lemmas 3.8 and 3.9 below are adapted from Kamihigashi and Roy (2003, Lemmas 3.6, 3.7); the proofs are omitted.

Lemma 3.8. Let $\left\{c_{t}, x_{t}\right\}$ be an optimal path. Let $t \in \mathbb{Z}_{+}$. If $c_{t}>0$ and $x_{t+2}>r_{+}\left(x_{t+1}\right)$, then

$$
1 \geq \delta f_{+}^{\prime}\left(x_{t+1}\right)
$$

If $x_{t+1}>r\left(x_{t}\right)$ and $c_{t+1}>0$, then

$$
1 \leq \delta f_{-}^{\prime}\left(x_{t+1}\right)
$$

Lemma 3.9. If $x>0$ is a steady state, then $g(x)>0$ and

$$
\delta f_{+}^{\prime}(x) \leq 1 \leq \delta f_{-}^{\prime}(x)
$$

The following result provides a link between the generalized derivatives of $f$ and the monotonicity properties of $\gamma$.

Lemma 3.10. Let $0 \leq a<b$. (i) If $\delta f_{+}^{\prime}>1$ on $[a, b)$, $\gamma$ is strictly increasing on $[a, b]$. (ii) If $\delta f_{-}^{\prime}<1$ on $(a, b], \gamma$ is strictly decreasing on $[a, b]$.

Proof. Since $\gamma_{+}^{\prime}=\delta f_{+}^{\prime}-1$ and $\gamma_{-}^{\prime}=\delta f_{-}^{\prime}-1$, the lemma follows from Giorgi and Kolmósi (1992, Theorem 1.13).

\section{Monotonicity and Convergence}

In the case of strictly concave utility, optimal capital paths are known to be monotone (Kamihigashi and Roy, Lemma 3.2). As shown in Proposition 3.2, this property does not carry over to the linear utility case. The following result however shows that an optimal capital path is strictly monotone until it reaches a steady state. 
Proposition 4.1. Let $\left\{x_{t}\right\}$ be an optimal capital path. Suppose $\exists T \in \mathbb{N}, \forall t \leq$ $T, x_{t}$ is not a steady state. Then $\left\{x_{t}\right\}_{t=0}^{T+1}$ is strictly monotone.

Proof. Since $x_{0}$ is not a steady state, $x_{0} \neq x_{1}$. Assume $x_{0}<x_{1}$ without loss of generality. If $x_{1} \geq x_{2}$, then $x_{1}$ is a steady sate by Lemma 3.6(i), a contradiction. Thus $x_{1}<x_{2}$. By induction, $\forall t \leq T, x_{t}<x_{t+1}$.

It can be expected from Proposition 4.1 that an optimal capital path reaches a steady state either in finite time or asymptotically. The next result shows that an optimal capital path either (i) converges to zero or (ii) reaches a positive steady state in finite time and possibly jumps among different steady states afterwards. Let $S$ be the set of steady states.

Proposition 4.2. Let $\left\{x_{t}\right\}$ be an optimal capital path. Then one of the following holds:

$$
\begin{gathered}
\lim _{t \uparrow \infty} x_{t}=0 ; \\
\exists T \in \mathbb{Z}_{+}, \forall t \geq T, \quad x_{t} \in S .
\end{gathered}
$$

The proof appears in Subsection 4.1. Mitra and Ray (1984, Theorem 6.1) showed that every optimal capital path approaches the set of steady states asymptotically in the case of concave utility and reversible investment with a continuous production function. Proposition 4.2 offers a sharper characterization in the case of linear utility and irreversible investment with a generally discontinuous production function.

Proposition 4.2 shows that there are only two possibilities for the asymptotic behavior of an optimal capital path. One might wonder if the first case (4.1) could be combined into the second, but convergence to zero simply cannot occur in finite time if $r(x)$ is strictly increasing near $x=0$. Hence an additional assumption is needed to ensure that convergence to zero also occurs in finite time.

\subsection{Proof of Proposition 4.2}

Lemma 4.1. Let $x>0$ be such that $g_{-}(x) \leq 0 .{ }^{12}$ Then for every feasible capital path $\left\{x_{t}\right\}$ from $x_{0} \in[0, x), \forall t \in \mathbb{Z}_{+}, x_{t}<x$.

\footnotetext{
${ }^{12}$ By upper semicontinuity, $g_{-}(x) \leq g(x)\left(=g_{+}(x)\right)$.
} 
Proof. Since $x_{1} \leq f\left(x_{0}\right)<f_{-}(x), x_{1}-x<g_{-}(x) \leq 0$. By induction, $\forall t \in \mathbb{N}, x_{t}<x$.

Lemma 4.2. Let $x>0$ be such that $g_{-}(x) \leq 0$. Then $\exists \bar{x} \in(0, x), \forall y \in$ $[0, x), \bar{K}(y)<\bar{x}$.

Proof. Suppose $g(x)=g_{-}(x) \leq 0$. Then $\forall y \in F(x), y \leq x$ by Remark 2.1, and $x \notin K(x)$ by Lemma 3.9; thus $\bar{x} \equiv \bar{K}(x)<x$. Hence the conclusion holds by Lemma 3.5(ii).

Now suppose $g(x)>0$. Let $\tilde{f}$ be a production function satisfying Assumptions 2.2-2.4 such that $\forall y \in[0, x), \tilde{f}(y)=f(y)$ and $\tilde{f}(x)=f_{-}(x)$. Let $\tilde{K}$ be the corresponding policy correspondence. By Lemma 4.1, every feasible capital path $\left\{x_{t}\right\}$ from $y \in[0, x)$ satisfies $\forall t \in \mathbb{Z}_{+}, x_{t}<x$ for the original problem. This implies $\forall y \in[0, x), K(y)=\tilde{K}(y)$. Hence the argument in the previous case applies.

For the rest of the proof, we take an optimal capital path $\left\{x_{t}\right\}$ as given.

Lemma 4.3. Suppose there exists a subsequence $\left\{x_{t_{i}}\right\}_{i=1}^{\infty}$ of $\left\{x_{t}\right\}$ such that $x \equiv \lim _{i \uparrow \infty} x_{t_{i}}>0$. Then (i) $\overline{\lim }_{i \uparrow \infty} g\left(x_{t_{i}}\right)>0$. Furthermore, (ii) $\forall I \in$ $\mathbb{N}, \exists i \geq I, x \in F\left(x_{t_{i}}\right)$.

Proof. Taking a further subsequence, we may assume $\left\{x_{t_{i}}\right\}$ is monotone. To show (i), suppose

$$
\lim _{i \uparrow \infty} g\left(x_{t_{i}}\right) \leq 0
$$

where the limit exists by monotonicity. Since $g_{-}(x) \leq \lim _{i \uparrow \infty} g\left(x_{t_{i}}\right) \leq 0$, $\left\{x_{t_{i}}\right\}$ is nonincreasing by Lemma 4.2. Applying Lemma 3.7 to each pair of the form $\left(x_{t_{i}}, x_{t_{i+1}}\right)$, we see that there is a nonincreasing optimal capital path $\left\{y_{t}\right\}$ from $x_{t_{1}}$ with $\lim _{t \uparrow \infty} y_{t}=x$. If $\exists T \in \mathbb{Z}_{+}, y_{t}=x$, then $x$ is a steady state and $g(x) \leq 0$ by (4.3), contradicting Lemma 3.9. It follows that

$$
\forall t \in \mathbb{Z}_{+}, \quad x<y_{t} .
$$

We have

$$
\lim _{t \uparrow \infty}\left[f\left(y_{t}\right)-y_{t+1}\right]=\lim _{t \uparrow \infty}\left[f\left(y_{t}\right)-y_{t}\right]=\lim _{t \uparrow \infty} g\left(y_{t}\right) \leq 0,
$$


where the last inequality holds by (4.3). Let $a=-\lim _{t \uparrow \infty} g\left(y_{t}\right)$. Suppose $a>0$. Let $b \in(0, a)$. Then $y_{t+1}-y_{t} \leq f\left(y_{t}\right)-y_{t}<-b$ for large $t,{ }^{13}$ which implies $\lim _{t \uparrow \infty} y_{t}=-\infty$, a contradiction. Thus

$$
0=\lim _{t \uparrow \infty} g\left(y_{t}\right)=g_{+}(x)=f(x)-x,
$$

where the last equality holds by upper semicontinuity. By Fatou's lemma,

$$
\begin{aligned}
\varlimsup_{t \uparrow \infty} v\left(y_{t}\right) & =\varlimsup_{t \uparrow \infty} \sum_{i=0}^{\infty} \delta^{i}\left[f\left(y_{t+i}\right)-y_{t+i+1}\right] \\
& \leq \sum_{i=0}^{\infty} \delta^{i} \varlimsup_{t \uparrow \infty}\left[f\left(y_{t+i}\right)-y_{t+i+1}\right] \leq 0,
\end{aligned}
$$

where the last inequality uses (4.5). On the other hand, by (3.5), (4.4), (4.6), and Assumption 2.3(ii).

$$
\begin{aligned}
\varlimsup_{t \uparrow \infty} v\left(y_{t}\right) & \geq \varlimsup_{t \uparrow \infty}\left[f\left(y_{t}\right)-r\left(y_{t}\right)\right] \\
& =f(x)-r_{+}(x)=x-r_{+}(x)>0,
\end{aligned}
$$

contradicting (4.7) and (4.8).

To show (ii), suppose $\exists I \in \mathbb{N}, \forall i \geq I, x \notin F\left(x_{t_{i}}\right)$. Since $r_{+}(x)<x$ by Assumption 2.3(ii), $r\left(x_{t_{i}}\right)<x$ for large $i$. Thus $x>f\left(x_{t_{i}}\right)$ for large $i$. But then $\overline{\lim }_{i \uparrow \infty} g\left(x_{t_{i}}\right)=\varlimsup_{\lim _{i \uparrow \infty}} f\left(x_{t_{i}}\right)-x \leq 0$, contradicting part (i).

Lemma 4.4. If $g\left(x_{t}\right) \leq 0$ infinitely often, then $\lim _{t \uparrow \infty} x_{t}=0$.

Proof. Let $\left\{x_{t_{i}}\right\}_{i=1}^{\infty}$ be a subsequence of $\left\{x_{t}\right\}$ such that $\forall i \in \mathbb{N}, g\left(x_{t_{i}}\right) \leq 0$. Taking a further subsequence, we may assume $\left\{x_{t_{i}}\right\}$ is convergent. Since $\varlimsup_{i \uparrow \infty} g\left(x_{t_{i}}\right) \leq 0, \lim _{t \uparrow \infty} x_{t}=0$ by Lemma 4.3(i). By Remark 2.1, $\forall i \in$ $\mathbb{N}, \forall t \geq t_{i}, x_{t} \leq x_{t_{i}}$. Hence $\lim _{t \uparrow \infty} x_{t}=0$.

Lemma 4.5. Suppose $\forall t \in \mathbb{Z}_{+}, g\left(x_{t}\right) \geq 0$. Then $\lambda\left(x_{t}\right)$ is nondecreasing in $t$.

Proof. Let $t \in \mathbb{Z}_{+}$. Since $g\left(x_{t}\right) \geq 0, x_{t} \in F\left(x_{t}\right)$. Thus by Lemma 3.4, $\lambda\left(x_{t}\right) \leq \lambda\left(x_{t+1}\right)$.

\footnotetext{
13 "For large $t$ " means "for $t \in \mathbb{Z}_{+}$sufficiently large." Similar conventions apply to similar expressions.
} 
Lemma 4.6. Suppose $\overline{\lim }_{t \uparrow \infty} x_{t}>0$. Then $\exists T \geq 0, \forall t \geq T$, (i) $\lambda\left(x_{t+1}\right)=$ $\overline{\lim }_{t \uparrow \infty} \lambda\left(x_{t}\right) \equiv \bar{\lambda}$ and (ii) $x_{t+1} \in S$.

Proof. By Lemma 4.4, $g\left(x_{t}\right)>0$ for large $t$. Hence we may assume $\forall t \in$ $\mathbb{Z}_{+}, g\left(x_{t}\right)>0$ without loss of generality. By Lemma $4.5, \lambda\left(x_{t}\right)$ is nondecreasing in $t$. Since $\overline{\lim }_{t \uparrow \infty} x_{t}>0$, there is a subsequence $\left\{x_{t_{i}}\right\}$ of $\left\{x_{t}\right\}$ with $x \equiv \lim _{t \uparrow \infty} x_{t_{i}}>0$. By monotonicity and upper semicontinuity,

$$
\bar{\lambda}=\sup _{t \in \mathbb{Z}_{+}} \lambda\left(x_{t}\right)=\lim _{t \uparrow \infty} \lambda\left(x_{t}\right) \leq \lambda(x) .
$$

By Lemma 4.3(ii), there is $i \in \mathbb{N}$ with $x \in F\left(x_{t_{i}}\right)$. Let $T=t_{i}$. Then by (4.11), Lemma 3.4, and monotonicity of $\lambda\left(x_{t}\right), \forall t \geq T, \bar{\lambda} \leq \lambda(x) \leq \lambda\left(x_{T+1}\right) \leq$ $\lambda\left(x_{t+1}\right) \leq \bar{\lambda}$; thus (i) follows. To see (ii), let $t \geq T$. Suppose $x_{t+1} \notin S$. Then by Corollary 3.1, $\lambda\left(x_{t+2}\right)>\lambda\left(x_{t+1}\right)$, contradicting part (i).

Proposition 4.2 now follows from Lemma 4.6.

\section{Survival, Extinction, and a Minimum Safe Standard of Conservation}

This section gives conditions for survival, extinction, and the existence of a "minimum safe standard of conservation." Our first result here provides a condition for survival, i.e., for an optimal capital path to be bounded away from zero. Together with Proposition 4.2, it extends Majumdar and Mitra (1983, Propositions 2, 3) to our general setting.

Proposition 5.1. Suppose

$$
\exists \bar{x}>0, \forall x \in(0, \bar{x}), \quad \delta f_{+}^{\prime}(x)>1 .
$$

Then every optimal capital path $\left\{x_{t}\right\}$ from $x_{0}>0$ satisfies $x_{t} \geq \bar{x}$ for large $t$.

Proof. By (5.1) and Lemma 3.9, there is no steady state in $(0, \bar{x})$. Let $x \in$ $(0, \bar{x})$. We claim $\underline{K}(x)>x$. Suppose $\underline{K}(x) \leq x$. Since $x$ is not a steady state, $\underline{K}(x)<x$. By Lemma 3.5(ii), there is an optimal capital path $\left\{x_{t}\right\}$ from $x$ with $\forall t \in \mathbb{N}, x_{t} \leq \underline{K}(x)<x$. By Lemma 3.10, $\gamma$ is strictly increasing on $[0, \bar{x}],{ }^{14}$ so $\forall t \in \mathbb{Z}_{+}, \gamma\left(x_{t+1}\right)<\gamma\left(x_{0}\right)$, contradicting Lemma 3.2. We have shown that $\forall x \in(0, \bar{x}), \underline{K}(x)>x$. Since there is no steady state in $(0, \bar{x})$, the conclusion holds by Proposition 4.2.

\footnotetext{
${ }^{14}$ The origin is included since $\gamma$ is continuous at 0 by Assumption 2.2.
} 
The next result offers a condition for global extinction, i.e., for every optimal capital path to converge to zero. It extends Majumdar and Mitra (1983, Proposition 1) to our general setting.

Proposition 5.2. Suppose

$$
\forall x>0, \quad \delta f_{-}^{\prime}(x)<1 .
$$

Then every optimal capital path converges to zero.

Proof. By (5.2) and Lemma 3.10, $\gamma$ is strictly decreasing on $[0, \infty)$. Thus every optimal capital path is nonincreasing by Lemma 3.3. It converges to zero by Proposition 4.2 since there is no steady state in $(0, \infty)$ by $(5.2)$ and Lemma 3.9.

The next result gives a condition for local extinction, i.e., for any optimal capital path starting below a certain level to converge to zero. It generalizes Majumdar and Mitra (1983, Lemma 4) to our general setting.

Proposition 5.3. Suppose

$$
\varlimsup_{x \downarrow 0} \delta f_{-}^{\prime}(x)<1
$$

Then $\exists \bar{x}>0$, any optimal capital path from $x \in(0, \bar{x}]$ converges to zero.

Before proving Proposition 5.3, let us show the existence of a "minimum safe standard of conservation" (e.g., Clark, 1971) under an additional condition. The following result along with Proposition 4.2 extends the analysis of Majumdar and Mitra (1983, Section 3c) to our general setting.

Proposition 5.4. Assume (5.3). Suppose $\max _{x \geq 0} \gamma(x)>0$. Then there exists $\underline{x}>0$ such that from any $x \in(0, \underline{x})$ there exists an optimal capital path converging to zero, and from any $x>\underline{x}$ there exists no optimal capital path converging to zero.

Proof. Let $\underline{x}=\sup \{x>0 \mid$ there is an optimal capital path from $x$ converging to zero $\}$. By Proposition 5.3, $\underline{x}>0$. Note from Lemma 3.5 that if there is an optimal capital path from $x>0$ converging to zero, then from any $x^{\prime} \in(0, x)$ there is an optimal capital path converging to zero. It follows that $\underline{x}$ has the desired property except for its finiteness. Since $\max _{x \geq 0} \gamma(x)>0$, there exists a steady state $\tilde{x}>0$ by Proposition 3.1. Since no optimal capital path from $\tilde{x}$ converges to zero by Lemma 3.3, we obtain $\underline{x} \leq \tilde{x}$. 


\subsection{Proof of Proposition 5.3}

Note that (5.3) implies

$$
\exists z>0, \exists \epsilon>0, \forall x \in(0, z], \quad \delta f_{-}^{\prime}(x)<1-\epsilon .
$$

Lemma 5.1. Under (5.4), $\exists \bar{x} \in(0, z]$, any optimal capital path from $x \in$ $(0, \bar{x}]$ is bounded above by $z$, where $z$ is given by (5.4).

Proof. If $\exists x \in(0, z], g(x) \leq 0$, then the conclusion follows from Remark 2.1. So suppose

$$
\forall x \in(0, z], \quad g(x)>0 .
$$

Suppose the conclusion is false. Then $\forall \bar{x} \in(0, z], \exists x \in(0, \bar{x}]$, there is an optimal capital path $\left\{x_{t}\right\}$ from $x$ with the property that $\exists t \in \mathbb{N}, x_{t}>z$. By Lemma 3.5, this means that $\forall x \in(0, z]$, there is an optimal capital path with the same property. For $i \in \mathbb{N}$, define $x^{i}=f^{-i}(z)$; this is well-defined since $f$ is strictly increasing, (5.4) implies that $f$ is continuous on $[0, z]$, and $f(z)>z$ by (5.5). By definition and (5.5),

$$
\forall i \in \mathbb{N}, \quad \text { (a) } f^{i}\left(x^{i}\right)=z, \quad \text { (b) } z>x^{i}=f\left(x^{i+1}\right)>x^{i+1} .
$$

Fix $i \in \mathbb{N}$ for the moment. Let $\left\{x_{t}^{i}\right\}$ be an optimal capital path from $x^{i}$ such that $\exists t \in \mathbb{N}, x_{t}^{i}>z$. Let $s \in \mathbb{N}$ be the first $t \in \mathbb{N}$ with $x_{t}^{i}>z$. Then

$$
\text { (a) } s>i, \quad \text { (b) } z<x_{s}^{i} \leq f(z) \text {. }
$$

Since there is no steady state in $(0, z]$ by (5.4) and Lemma 3.9, $\left\{x_{t}^{i}\right\}_{t=0}^{s}$ is strictly increasing by Proposition 4.1. Thus $\forall t \in\{0,1, \ldots, s-1\}, x_{t+1}^{i}>$ $x_{t}^{i}>r\left(x_{t}^{i}\right)$, where the last inequality holds by Assumption 2.3(ii). Hence by Lemma 3.8 and (5.4),

$$
\left.\forall t \in\{1, \ldots, s-1\}, \quad x_{t+1}^{i}=f\left(x_{t}^{i}\right) \quad \text { (i.e., } c_{t}^{i}=0\right) .
$$

Since the stationary path from $x^{i}$ is feasible by (5.5), it follows that

$$
\frac{g\left(x^{i}\right)}{1-\delta} \leq v\left(x^{i}\right)=f\left(x^{i}\right)-x_{1}^{i}+\delta^{s} v\left(x_{s}\right)<g\left(x^{i}\right)+\delta^{s} \bar{v},
$$

where $\bar{v}=\max _{x \in[z, f(z)]} v(x)$, the equality follows from (5.8), and the last inequality holds by (5.7)(b) since $x_{1}^{i}>x^{i}$. From (5.5), (5.9), and (5.7)(a),

$$
0<\frac{\delta g\left(x^{i}\right)}{1-\delta}<\delta^{s} \bar{v} \leq \delta^{i+1} \bar{v}
$$


Thus $\delta^{-i} g\left(x^{i}\right) \leq(1-\delta) \bar{v}$. Since the right-hand side is finite and does not depend on $i$, to obtain a contradiction it suffices to show

$$
\lim _{i \uparrow \infty} \delta^{-i} g\left(x^{i}\right)=\infty
$$

Note that $\forall i \in \mathbb{N}$,

$$
\begin{aligned}
\frac{\delta^{-i} g\left(x^{i}\right)}{\delta^{-(i+1)} g\left(x^{i+1}\right)} & =\delta \frac{f\left(x^{i}\right)-x^{i}}{f\left(x^{i+1}\right)-x^{i+1}} \\
& =\delta \frac{f\left(x^{i}\right)-f\left(x^{i+1}\right)}{x^{i}-x^{i+1}} \\
& \leq \delta f_{-}^{\prime}\left(\tilde{x}^{i}\right) \leq 1-\epsilon,
\end{aligned}
$$

where (5.13) uses (5.6)(b), the first inequality in (5.14) holds for some $\tilde{x}^{i} \in$ $\left[x^{i+1}, x^{i}\right]$ by the generalized mean value theorem (Giorgi and Kolmósi, 1992, Theorem 1.8), and the last inequality holds by (5.4). Now (5.11) follows.

To complete the proof of Proposition 5.3, let $\bar{x}$ be as given by Lemma 5.1. Since there is no steady state in $(0, z]$ by $(5.4)$ and Lemma 3.9, any optimal capital path from $x \in(0, \bar{x}]$ converges to zero by Proposition 4.2 .

\section{Turnpike Properties}

This section shows that for any $\epsilon>0$, for $\delta$ close to one, any optimal capital path from $x_{0} \geq \epsilon$ "converges" to a small neighborhood of the golden rule capital stock. We start by assuming that maximum sustainable consumption is strictly positive.

Assumption 6.1. $g^{*} \equiv \max _{x \geq 0} g(x)>0$.

If $g^{*} \leq 0$, extinction occurs from every initial capital stock by Lemma 3.9 and Proposition 4.2. Assumption 6.1 rules out this trivial case.

Assumption 6.2. There exists a unique $x^{*} \geq 0$ such that $g\left(x^{*}\right)=g^{*} \cdot{ }^{15}$

We call $x^{*}$ the golden rule capital stock. The following is our last assumption.

\footnotetext{
${ }^{15}$ Along the lines of Kamihigashi and Roy (2003, Section 8) it is possible to deal with cases in which there are more than one capital stock at which $g(x)$ is maximized.
} 
Assumption 6.3. $\forall x \in\left(0, x^{*}\right], g_{-}(x)>0$.

If $g_{-}(x) \leq 0$ for some $x \in\left(0, x^{*}\right.$, no feasible capital path from $x_{0} \in(0, x)$ can reach $x^{*}$ by Lemma 4.1. Assumption 6.3 ensures that it is possible to reach $x^{*}$ from any $x \in\left(0, x^{*}\right]$. We are now ready to state the main result of this section.

Proposition 6.1. Let $\epsilon^{*} \in\left(0, x^{*}\right)$ and $\epsilon \in\left(0, x^{*}-\epsilon^{*}\right)$. Then for $\delta$ close to one, any optimal capital path $\left\{x_{t}\right\}$ from $x_{0} \in[\epsilon, \infty)$ satisfies

$$
\exists T \in \mathbb{Z}_{+}, \forall t \geq T, \quad x_{t} \in\left(x^{*}-\epsilon^{*}, x^{*}+\epsilon^{*}\right) .
$$

Before proving this result, let us show a simple consequence that extends Majumdar and Nermuth (1982, Theorem 3.4) to the case of linear utility. ${ }^{16}$

Corollary 6.1. Let $\epsilon^{*} \in\left(0, x^{*}\right)$. Suppose

$$
\frac{\lim }{x \downarrow 0} f_{+}^{\prime}(x)>1 .
$$

Suppose there exists a unique steady state $x(\delta)$ in $\left(x^{*}-\epsilon^{*}, x^{*}+\epsilon^{*}\right)$ for $\delta$ close to one. Then for $\delta$ close to one, every optimal capital path $\left\{x_{t}\right\}$ from $x_{0} \in(0, \infty)$ converges to $x(\delta)$.

Proof. By (6.2), $\exists \mu>1, \exists \bar{x}>0, \forall x \in(0, \bar{x}), f_{+}^{\prime}(x)>\mu$. Hence for $\delta$ close to one, $\forall x \in(0, \bar{x}), \delta f_{+}^{\prime}(x)>1$. Thus the conclusion follows from Propositions 5.1, 6.1 (with $\epsilon=\bar{x}$ ), and 4.2.

\subsection{Proof of Proposition 6.1}

Lemma 6.1. $\forall \epsilon \in\left(0, x^{*}-\epsilon^{*}\right)$, for $\delta$ close to one, there exists no steady state in $\left[\epsilon, x^{*}-\epsilon^{*}\right]$.

Proof. It is easy to see that $\exists T \in \mathbb{N}, \forall x_{0} \in\left[\epsilon, x^{*}-\epsilon^{*}\right]$, there is a feasible capital path from $x_{0}$ such that $\forall t \geq T, x_{t}=x^{*} \cdot{ }^{17}$ Let $\bar{g}=\max _{y \in\left[\epsilon, x^{*}-\epsilon^{*}\right]} g(y)$. By Assumption 6.2, for $\delta$ close to one, $\bar{g} /(1-\delta)<\delta^{T} g^{*} /(1-\delta)$, so the stationary path from any $x \in\left[\epsilon, x^{*}-\epsilon^{*}\right]$ is not optimal.

\footnotetext{
${ }^{16}$ For extensions of their result in the case of strictly concave utility, see Kamihigashi and Roy (2003, Section 8).

${ }^{17}$ Let $T \in \mathbb{N}$ be such that there is a feasible capital path from $\epsilon$ with $\forall t \geq T, x_{t}=x^{*}$. This $T$ works for all $x_{0} \in\left[\epsilon, x^{*}-\epsilon^{*}\right]$.
} 
Lemma 6.2. For $\delta$ close to one, there exists no steady state in $\left[x^{*}+\epsilon^{*}, \infty\right)$.

Proof. By Assumption 2.4, there is no steady state in $[\hat{x}, \infty)$ for any $\delta \in$ $(0,1)$. The argument of the proof of Lemma 6.1 shows that there is no steady state in $\left[x^{*}+\epsilon, \hat{x}\right]$ for $\delta$ close to one.

Lemma 6.3. Let $x \in\left(0, x^{*}-\epsilon^{*}\right)$ be such that

$$
f(x)<g^{*}{ }^{18}
$$

Then for $\delta$ close to one, $\underline{K}(x)>x .^{19}$

Proof. Let $T \in \mathbb{N}$ be such that there is a feasible capital path from $x$ with $\forall t \geq T, x_{t}=x^{*}$. Then

$$
\forall \delta \in(0,1), \quad v(x) \geq \delta^{T} g^{*} /(1-\delta) .
$$

Let $\delta \in(0,1)$ be such that

$$
f(x)<\delta^{T} g^{*}
$$

This inequality holds for $\delta$ close to one by (6.3). Suppose $\underline{K}(x) \leq x$. Then by Lemma $3.5(\mathrm{ii})$, there is an optimal capital path $\left\{x_{t}\right\}$ from $x$ such that $\forall t \in \mathbb{Z}_{+}, x_{t} \leq x$. Hence

$$
v(x)=\sum_{t=0}^{\infty} \delta^{t}\left[f\left(x_{t}\right)-x_{t+1}\right] \leq f(x) /(1-\delta),
$$

which together with (6.5) contradicts (6.4).

Let us now complete the proof of Proposition 6.1. If $f(\epsilon) \geq g^{*}$, make $\epsilon$ smaller so that $f(\epsilon)<g^{*}$. From Lemmas 6.1-6.3, for $\delta$ close to one, there is no steady state in $\left[\epsilon, x^{*}-\epsilon^{*}\right] \cup\left[x^{*}+\epsilon^{*}, \infty\right)$, and $\underline{K}(\epsilon)>\epsilon$. Hence any optimal capital path $\left\{x_{t}\right\}$ from $x_{0} \geq \epsilon$ satisfies $\forall t \in \mathbb{Z}_{+}, x_{t} \geq \underline{K}(\epsilon)>\epsilon$ by Lemma 3.5(ii), and thus satisfies (6.1) by Proposition 4.2.

\footnotetext{
${ }^{18}$ Such $x$ exists since $\lim _{x \downarrow 0} f(x)=0$ by Assumption 2.2 and $g^{*}>0$ by Assumption 6.1.

${ }^{19}$ For simplicity, we do not express the dependence of $K$ and $v$ on $\delta$.
} 


\section{References}

Clark, C.W., 1971, "Economically Optimal Policies for the Utilization of Biologically Renewable Resources," Mathematical Biosciences 12, 245-260.

Clark, C.W., 1990, Mathematical Bioeconomics: The Optimal Management of Renewable Resources, Second Edition. John Wiley \& Sons: New York.

Dechert, R., and K. Nishimura, 1983, "A Complete Characterization of Optimal Growth Paths in an Aggregated Model with a Non-Concave Production Function," Journal of Economic Theory 31, 332-354.

Ekeland, I., and J.A. Scheinkman, 1986, "Transversality Conditions for Some Infinite Horizon Discrete Time Optimization Problems," Mathematics of Operations Research 11, 216-229.

Giogi, G., and S. Komlósi, 1992, "Dini Derivatives in Optimization - Part I," Rivista di Matematica per le Scienze Economiche e Sociali 15, 3-30.

Kamihigashi, T., 1999, "Chaotic Dynamics in Quasi-Static Systems: Theory and Applications," Journal of Mathematical Economics 31, 183-214.

Kamihigashi, T., and S. Roy, 2003, "A Nonsmooth, Nonconvex Model of Optimal Growth," RIEB Discussion Paper 158, RIEB, Kobe University. (http://www.rieb.kobe-u.ac.jp/academic/ra/dp/English/dp158.pdf)

Majumdar, M., and T. Mitra, 1982, "Intertemporal Allocation with a NonConvex Technology: The Aggregative Framework," Journal of Economic Theory 27, 101-136

Majumdar, M., and T. Mitra, 1983, "Dynamic Optimization with a Non-Convex Technology: The Case of a Linear Objective Function," Review of Economic Studies 50, 143-151

Majumdar, M., and M. Nermuth, 1982, "Dynamic Optimization in Non-Convex Models with Irreversible Investment: Monotonicity and Turnpike Results," Journal of Economics 42, 339-362.

Malinvaud, E., 1965, "Croissances Optimales dan un Modele Macroeconomique," in The Econometric Approach to Development Planning. Pontificia Academia Scientiarum: Amsterdam.

McKenzie, L.W., 1986, "Optimal Economic Growth, Turnpike Theorems and Comparative Dynamics," in Handbook of Mathematical Economics, Vol. III, ed. by K.J. Arrow and M.D. Intrilligator. Amsterdam: NorthHolland, pp. 1281-1355. 
Mitra, T. and D. Ray, 1984, "Dynamic Optimization on a Non-Convex Feasible Set: Some General Results for Non-Smooth Technologies," Journal of Economics 44, 151-174.

Shell, K., 1967, "Optimal Programs of Capital Accumulation for an Economy in which There is Exogenous Technical Change," in Essays on the Theory of Optimal Economic Growth, K. Shell, ed. MIT Press: Cambridge, MA.

Skiba, A.K., 1978, "Optimal Growth with a Convex-Concave Production Function," Econometrica 46, 527-39.

Spence, M., and D. Starrett, 1973, "Most Rapid Approach Paths in Accumulation Problems," International Economic Review 16, 388-403.

Srinivasan, T.N., 1964, "Optimal Savings in a Two-Sector Model of Growth," Econometrica 32, 358-73.

Topkis, D.M., 1978, "Minimizing a Submodular Function on a Lattice," Operations Research 26, 305-321.

Uzawa, H., 1964, "Optimal Growth in a Two-Sector Model of Capital Accumulation," Review of Economic Studies 31, 1-24. 